\title{
Humoral response and breakthrough infections with SARS-CoV-2 B.1.617.2 variant in vaccinated maintenance hemodialysis patients
}

\author{
Ori Wand ${ }^{1,2} \cdot$ Naomi Nacasch $^{2,3} \cdot$ Ayman Fadeela $^{4} \cdot$ Moshe Shashar $^{5,6} \cdot$ Ayelet Grupper $^{2,7} \cdot$ Sydney Benchetrit ${ }^{2,3}$. \\ Daniel Erez ${ }^{2,8} \cdot$ Pnina Shitrit ${ }^{2,9} \cdot$ Keren Cohen-Hagai ${ }^{2,3}$ (1)
}

Received: 25 October 2021 / Accepted: 1 January 2022 / Published online: 17 February 2022

(c) The Author(s) under exclusive licence to Italian Society of Nephrology 2022

\begin{abstract}
Introduction Breakthrough COVID-19 may occur in vaccinated people, and may result from declining vaccine effectiveness or highly transmittable SARS-CoV-2 variants, such as the B.167.2 (delta) variant. We investigated risk factors and outcomes for infection with the delta variant among vaccinated hemodialysis patients.

Methods Patients on maintenance hemodialysis who received two doses of the BNT162b2 (Pfizer-BioNTech) vaccine were analysed according to having developed COVID-19 (study group) or not (control group), in a retrospective, observational, comparative study. We compared risk-factors for developing breakthrough COVID-19 and assessed clinical outcomes, including 30-day mortality rates.

Results Twenty-four cases of breakthrough SARS-CoV-2 infection were compared to 91 controls without infection. Breakthrough infection was associated with chronic immunosuppressive treatment, hematological malignancies, and low antibody levels against SARS-CoV-2 spike protein. All COVID-19 cases occurred at least 5 months after vaccination, and most were caused by the B.1.617.2 variant (at least 23/24 cases). COVID-19 was categorized as severe or critical disease in 11/24 patients (46\%), and 54\% required hospitalization and COVID-19-directed treatment. The source of infection was nosocomial in $6 / 24$ cases (25\%), and healthcare-related in $3 / 24(12.5 \%)$. Mortality rate was $21 \%$. Overall mortality was significantly higher in patients who developed COVID-19 than in controls (odds ratio for all-cause mortality 7.6, 95\% CI $1.4-41, \mathrm{p}=0.002)$.
\end{abstract}

Keren Cohen-Hagai

keren.cohen@ clalit.org.il

1 Department of Pulmonology, Meir Medical Center, Kfar Saba, Israel

2 Sackler Faculty of Medicine, Tel Aviv University, Tel Aviv, Israel

3 Department of Nephrology and Hypertension, Meir Medical Center, Kfar Saba, Israel

4 Corona and Respiratory Viruses Laboratory, Meir Medical Center, Kfar Saba, Israel

5 Department of Nephrology and Hypertension, Laniado Hospital, Netanya, Israel

6 Ruth and Bruce Rappaport Faculty of Medicine, Technion, Haifa, Israel

7 Department of Nephrology and Hypertension, Tel Aviv Sourasky Medical Center Tel Aviv, Tel Aviv, Israel

8 Department of Internal Medicine D, Meir Medical Center, Kfar Saba, Israel

9 Infection Control Unit, Meir Medical Center, Kfar Saba, Israel 
Conclusions Breakthrough COVID-19 with the B.1.617.2 variant can occur in vaccinated hemodialysis patients and is associated with immunosuppression and weaker humoral response to vaccination. Infections may be nosocomial and result in significant morbidity and mortality.

\section{Graphical abstract}

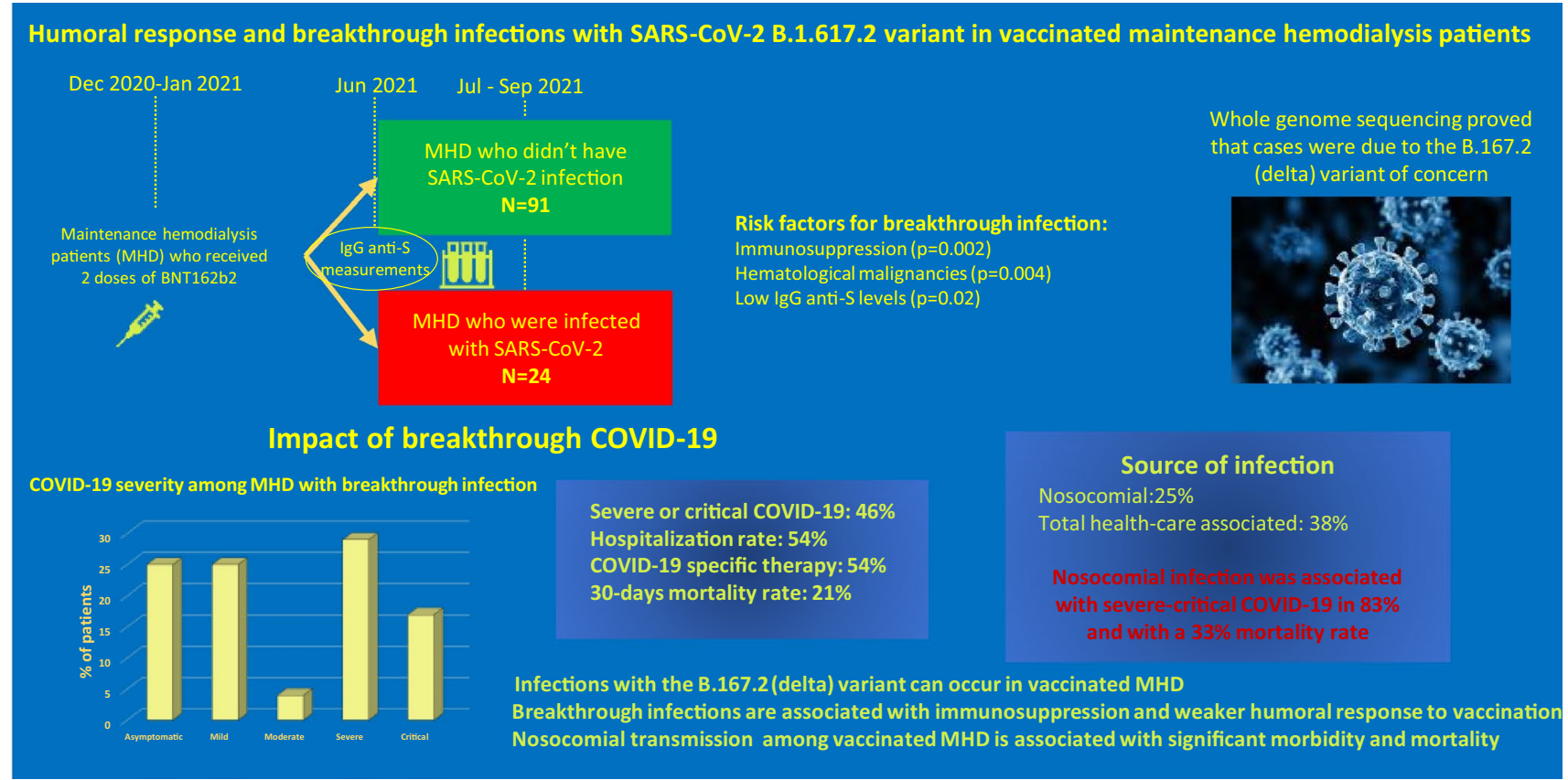

Keywords Breakthrough COVID-19 infection $\cdot$ SARS-CoV-2 variant $\cdot$ BNT162b2 vaccine $\cdot$ Maintenance hemodialysis

\section{Introduction}

Highly effective vaccines against severe acute respiratory syndrome virus 2 (SARS-CoV-2) have been developed and administered worldwide. Protection from coronavirus disease 2019 (COVID-19), however, is not absolute. Concerns regarding breakthrough COVID-19 in vaccinated patients are increasing, as vaccine efficacy appears to gradually decline in the months following vaccination $[1,2]$. The emergence of highly infectious variants and especially the predominance of the B.1.617.2 (delta) variant, escalate these concerns [3, 4].

End-stage kidney disease requiring maintenance hemodialysis (MHD) is a risk-factor for severe COVID-19 infection and mortality [5]. Vaccinated patients on MHD may be more susceptible to breakthrough COVID-19 because of impaired immune systems and increased exposure. While most MHD patients develop an immune response following messenger RNA (mRNA) vaccination [6], this response is weaker than in the healthy population [7], and may diminish over time [8].

We report on breakthrough COVID-19 with variant B.1.617.2 in vaccinated patients on MHD, including an association with humoral response to vaccination and other clinical variables.

\section{Methods}

\section{Study design}

This retrospective, observational study was conducted at Meir Medical Center, Kfar Saba, Israel, from June 1 to September 30, 2021 and included patients with end-stage kidney disease on MHD.

Results are reported according to the STROBE statement guidelines.

Preventive measures that were applied to mitigate COVID-19 spread in the hemodialysis unit are detailed in S1. Supplemental Methods Section.

\section{Participants}

Participants included patients $\geq 18$ years of age on MHD in our institution. MHD was defined as at least 3 months of hemodialysis prior to the study period. 
All participants received 2 doses of the BNT162b2 (Pfizer-BioNTech) vaccine at a 21-day interval. Patients were vaccinated as part of a national vaccination strategy prioritizing MHD patients, which began in December 2020.

\section{Study groups}

Participants were categorized to a study group that included MHD patients who were infected with SARS-CoV-2 during the study period and at least one week after the second vaccine dose. Some patients in this group had SARS-CoV-2 antibody measurements taken 6 months after vaccination and before their infection, as part of a different study [8].

Vaccinated MHD patients without evidence of SARSCoV-2 infection, who had SARS-CoV-2 antibody measurements 6 months after vaccination served as the control group. Patients without breakthrough infection who did not have antibody measurements were excluded.

This analysis includes data regarding two doses of the BNT162b2 vaccine. A third vaccine dose was recommended and available in Israel for high-risk individuals, including MHD patients, beginning in July 2021. Data of patients who received a third dose were included only from the second dose until the third dose.

\section{Diagnosis of SARS-CoV-2 infection}

SARS-CoV-2 infection was diagnosed either by positive real-time reverse-transcriptase polymerase chain reaction (RT-PCR) or by the presence of anti-nucleocapsid IgG antibodies (anti-N-Ab). RT-PCR result from nasopharyngeal swabs was considered positive if the cycle threshold was 40 or less.

SARS-CoV-2 anti-N-Ab were measured in all study participants in September 2021, in order to exclude asymptomatic infection. Titers higher than 1 index were considered positive. Anti-N-Ab were measured using the Architect SARS-CoV-2 IgG nucleocapsid protein assay (Abbot, Abbot Park, IL).

An experienced infection control physician (P.S.) reviewed the medical records of individual cases to determine where SARS-CoV-2 infection was acquired. Patients whose symptom onset and first positive RT-PCR occurred on hospital day 7 or later, or that occurred within 14 days of hospital discharge were suspected to be nosocomial.

\section{Variables}

Clinical, laboratory and radiologic data were extracted from the patients' medical records. The day of first positive swab for SARS-CoV-2 served as day 0 of illness for the study.

Baseline clinical variables included age, sex, comorbidities, dialysis vintage and adequacy parameters, and baseline laboratory data. Laboratory test results collected up to a month before the diagnosis of COVID-19 or at the time of IgG anti-S measurements served as baseline for the study and control groups, respectively.

Variables related to COVID-19 included clinical findings at presentation (COVID-19 symptoms and vital signs), laboratory findings (blood count, chemistry results, C-reactive protein and clotting tests), radiology findings (from chest $\mathrm{X}$-rays and computerized tomography studies, when performed) and treatment given for COVID-19.

Levels of antibodies targeting SARS-CoV-2 spike protein (IgG anti-S) were measured using the Abbott AdviseDx SARS-CoV-2 IgG II Quant assay on an Architect i200SR analyzer. A cutoff of $\geq 50 \mathrm{AU} / \mathrm{ml}$ was considered a significant antibody response, as previously suggested [8].

\section{Viral whole genome sequencing}

Complete SARS-CoV-2 genomes were sequenced via next generation sequencing, as previously described [9].

\section{Outcome measures}

The primary outcome for the study group was 30-day mortality after COVID-19 infection. Secondary outcomes included mortality rates during follow-up, disease severity, and hospital admission rates.

Disease severity was ranked according to National Institutes of Health (NIH) guidelines as asymptomatic, mild, moderate (with clinical or radiographic evidence of lower respiratory tract disease and oxygen saturation $\geq 94 \%$ while breathing room air), severe (saturation $<94 \%$, respiratory rate $>30 / \mathrm{min}$, infiltrates over $50 \%$ of lung volume, or $\mathrm{PaO}_{2} /$ $\mathrm{FiO}_{2}$ ratio $<300$ ), or critical (individuals with respiratory failure requiring invasive or non-invasive ventilation, septic shock, or multiorgan dysfunction) [10].

To assess risk-factors for breakthrough COVID-19, we compared baseline clinical and laboratory parameters as well as baseline IgG anti-S levels between the study and control groups prior to COVID-19 infection.

\section{Statistical analysis}

Descriptive statistics are presented as means, medians or percentages with standard deviations and ranges, as appropriate. Comparison of variables between the two study groups was performed using t test, Mann-Whitney test, Fisher's exact test or chi-square test, as appropriate. Univariate and multivariate logistic regression models were applied to estimate the odds ratio (OR) for infection and mortality. Survival curves were obtained using the Kaplan-Meier method and compared using two-sided log rank statistics. $\mathrm{p}$ values $<0.05$ were considered statistically significant. 
Data were analyzed with SPSS, version 27 (IBM Corporation, Armonk, NY, USA).

\section{Ethical concerns}

The study was approved by the Ethics Committee and Institutional Review Board of Meir Medical Center (no. MMC 16-21). Patients with assessment of humoral response provided signed informed consent. The committee waived the requirement for other participants' informed consent due to the retrospective nature of the study. The study was performed in accordance with the Declaration of Helsinki and the Good Clinical Practice Guidelines.

\section{Results}

A total of 24 MHD patients had breakthrough SARS-CoV-2 infection despite 2 doses of the BNT162b2 vaccine (study group). The control group consisted of 91 MHD patients who were not infected and had IgG anti-S measurements. A flow-chart of participant recruitment is available in Supplementary Figure S2. COVID-19 was diagnosed by a positive RT-PCR in 23 patients. One additional case was diagnosed by conversion of the anti-N-Ab after the second vaccine dose. Compared to the control group, the study group contained more immunosuppressed patients (6/91 vs. 8/24, respectively; p0.002) and more with hematological malignancies ( $2 / 91$ vs. $4 / 24$, respectively; $p=0.004)$. Mean baseline white blood cell counts and absolute neutrophil counts were higher among the study group. Other baseline clinical characteristics and laboratory findings were similar between the groups (Table 1).

\section{Clinical risk factors for breakthrough SARS-CoV-2 infection}

In univariate analysis, chronic immunosuppressive therapy, hematologic malignancy and ischemic heart disease were associated with the risk of developing breakthrough SARS$\mathrm{CoV}-2$ infection. In multivariate analysis of comorbidities,
Table 1 Baseline clinical and laboratory characteristics of study groups

\begin{tabular}{|c|c|c|c|}
\hline Characteristic & Study group $(n=24)$ & Control group $(n=91)$ & $\mathrm{p}$ value \\
\hline Age (years) & $70.3 \pm 15$ & $70.9 \pm 14.3$ & 0.8 \\
\hline Male sex & $19(79.2)$ & $58(63.7)$ & 0.1 \\
\hline Dialysis vintage (months) & $29.6 \pm 19.3$ & $30.8 \pm 25.1$ & 0.8 \\
\hline Weight (kg) & $75.4 \pm 13.6$ & $77.8 \pm 19.9$ & 0.6 \\
\hline \multicolumn{4}{|l|}{ Comorbidities } \\
\hline Diabetes mellitus & $14(58.3)$ & $59(64.8)$ & 0.6 \\
\hline Hypertension & $20(83.3)$ & $73(80.2)$ & 0.7 \\
\hline Ischemic heart disease & $13(54.2)$ & $31(34.1)$ & 0.07 \\
\hline Heart failure & $12(50)$ & $29(31.9)$ & 0.09 \\
\hline Peripheral vascular disease & $2(8.3)$ & $9(9.9)$ & 0.8 \\
\hline Chronic lung disease & $3(12.5)$ & $12(13.2)$ & 0.9 \\
\hline Solid malignancy & $0(0 \%)$ & $3(3.3)$ & 0.4 \\
\hline Hematologic malignancy & $4(16.7)$ & $2(2.2)$ & 0.004 \\
\hline Chronic immunosuppressive therapy & $7(29.2)$ & $6(6.6)$ & 0.002 \\
\hline \multicolumn{4}{|l|}{ Baseline laboratory data } \\
\hline Creatinine (mg/dL) & $7.2 \pm 2.5$ & $7.1 \pm 2.3$ & 0.8 \\
\hline White blood cell count $(\mathrm{K} / \mu \mathrm{L})$ & $7.8 \pm 3.2$ & $6.3 \pm 1.9$ & 0.006 \\
\hline Absolute neutrophils $(\mathrm{K} / \mu \mathrm{L})$ & $5.3 \pm 2.5$ & $4.2 \pm 1.5$ & 0.006 \\
\hline Absolute lymphocytes $(\mathrm{K} / \mu \mathrm{L})$ & $1.4 \pm 0.5$ & $1.4 \pm 0.6$ & 0.7 \\
\hline Neutrophil to lymphocyte ratio & $4.4 \pm 2.8$ & $3.6 \pm 2$ & 0.1 \\
\hline Hemoglobin (g/dL) & $10.7 \pm 1.1$ & $10.6 \pm 1.2$ & 0.7 \\
\hline Albumin (g/dL) & $3.6 \pm 0.4$ & $3.6 \pm 0.4$ & 0.6 \\
\hline $\mathrm{Kt} / \mathrm{V}$ & $1.41 \pm 0.26$ & $1.28 \pm 0.22$ & 0.091 \\
\hline Urea reduction ratio & $69.6 \pm 5.4$ & $66.7 \pm 6.7$ & 0.203 \\
\hline Serum IgG S titer (AU/mL) & $89.1 \pm 114.5$ & $533.7 \pm 726.8$ & 0.111 \\
\hline
\end{tabular}

Data are presented as mean $\pm \mathrm{SD}$ or as absolute numbers $(\%)$. Laboratory tests were taken at dialysis initiation as part of routine follow-up of MHD patients

Bold values represent statistically significant results $p<0.05$ 
chronic immunosuppression remained the most significant predictor of COVID-19 (Table 2).

\section{Baseline humoral response and breakthrough infection}

Serological test results for IgG anti-S performed 6 months after the first vaccination dose (and before SARS-CoV-2 infection) were available for 7/24patients in the study group (29\%) and for all 91 in the control group. Baseline mean IgG anti-S levels were numerically lower in the study group than in controls $(89.1 \pm 114.5 \mathrm{AU} / \mathrm{ml}$ vs. $533.7 \pm 726.8 \mathrm{AU} /$ $\mathrm{ml}$, respectively, $\mathrm{p}=0.111$ ), despite comparable intervals from the second vaccine dose to IgG anti-S measurement (168.2 \pm 15 days vs. $158.5 \pm \pm 17.7$ days, $p=0.193$ ), Fig. 1 .

The proportion of patients with low IgG anti-S levels $(<50 \mathrm{AU} / \mathrm{ml})$ was significantly higher in the study group compared to the control group (4/7 (57.1\%) vs. $17 / 91$ $(18.7 \%)$, respectively, $\mathrm{p}=0.017)$. The OR for SARS-CoV-2 infection was 5.8 (95\% confidence interval (CI) 1.2-28.4) when the IgG anti-S level at baseline was $<50 \mathrm{AU} / \mathrm{ml}$.

\section{SARS-CoV-2 infection}

COVID-19 was diagnosed an average of $190.7 \pm 17.9$ days after the second vaccine dose (median 191, range 154-211). We did not find any cases of SARS-CoV-2 infection during the first 5 months following 2 doses of BNT162b2 vaccine in our dialysis unit (although we did find SARS-CoV-2 infection among unvaccinated patients).

Asymptomatic patients were diagnosed with either positive anti-N-Ab or positive RT-PCR taken after exposure/ close contact with a confirmed COVID-19 case. Universal screening in vaccinated patients was not part of the infection control policy in our medical center at the time of the study.

COVID-19 was asymptomatic in 7/24 MHD patients (29\%), including the patient who was diagnosed by a positive anti-N-Ab. The most common presenting symptoms were fever (58\%) and cough (54\%), Table 3.

There were no significant differences in the interval from vaccine, baseline IgG anti-S levels, age, comorbidities,

Table 2 Multivariate logistic regression analysis model for predictors of COVID-19 infection

\begin{tabular}{lllll}
\hline Variable & OR & \multicolumn{2}{l}{$95 \%$ CI } & p value \\
\cline { 3 - 4 } & & Lower & Upper & \\
\hline Sex (male) & 1.8 & 0.6 & 5.9 & 0.3 \\
Ischemic heart disease & 1.8 & 0.6 & 5 & 0.3 \\
Heart failure & 2.6 & 0.9 & 7.3 & 0.08 \\
Hematologic malignancy & 2.6 & 0.3 & 27.8 & 0.4 \\
Immunosuppressive therapy & 4.8 & 0.9 & 25.8 & 0.06 \\
\hline
\end{tabular}

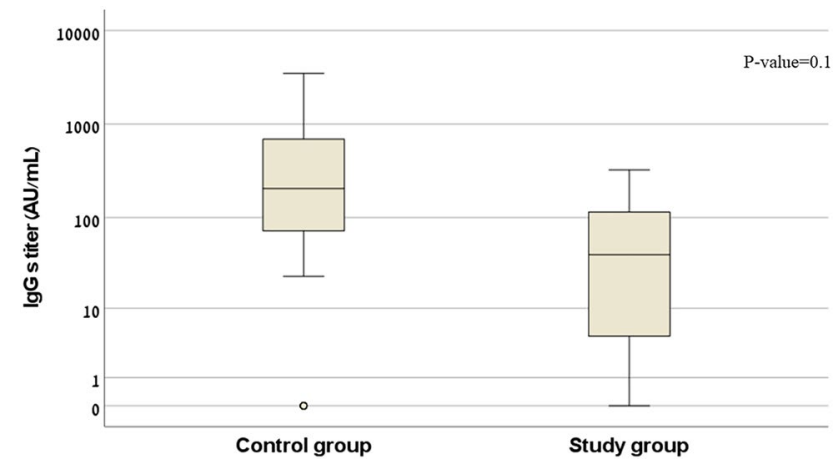

Fig. 1 Box plot of baseline IgG anti-S titer. Mean IgG anti-S level in the study group was $89.1 \pm 114.5 \mathrm{AU} / \mathrm{ml}$ vs. $533.7 \pm 726.8 \mathrm{AU} / \mathrm{ml}$ in the control group, $\mathrm{p}=0.1$. Median (range) was $40(0-324)$ and 204.5 $(0-3,496)$, respectively. IgG anti-S titer is shown in logarithmic scale. The medians are marked by horizontal lines inside the boxes. Error bars represent the range between minimum and maximum points in each group. IgG anti-S, antibodies targeting SARS-CoV-2 spike protein

dialysis vintage and adequacy among patients who had asymptomatic COVID-19 infection and those who were symptomatic.

COVID-19 disease was categorized as severe or critical in $11 / 24$ patients (46\%; Fig. 2). Patients who developed severe/critical COVID-19 were on average older than those who had milder disease $(77.6 \pm 8.9$ years vs. $64.7 \pm 16.1$, $\mathrm{p}=0.025)$. Other characteristics were similar between patients with severe-critical disease and those with milder COVID-19. Patients with severe/critical COVID19 had worse inflammatory markers at presentation,

Table 3 Clinical presentation and outcomes of COVID-19 breakthrough infection

\begin{tabular}{ll}
\hline Clinical manifestation & $\mathrm{N}(\%)$ \\
\hline Asymptomatic & $6(25)$ \\
Fever & $14(58.3)$ \\
Cough & $13(54.2)$ \\
Dyspnea & $7(29.2)$ \\
Loss of taste and smell & $1(4.2)$ \\
Gastrointestinal symptoms & 0 \\
Nausea & $2(8.3)$ \\
Fatigue & $6(25)$ \\
Laboratory tests at presentation & \\
White blood cell count $(\mathrm{K} / \mu \mathrm{L})$ & $6.7 \pm 4$ \\
Platelets $(\mathrm{K} / \mu \mathrm{L})$ & $169 \pm 52$ \\
Neutrophil to lymphocyte ratio & $6 \pm 3.9$ \\
C-reactive protein (mg/dL) & $6.7 \pm 5.9$ \\
Ferritin ( $\mu \mathrm{g} / \mathrm{L})$ & $785 \pm 516$ \\
Albumin $(\mathrm{g} / \mathrm{dl})$ & $3.3 \pm 0.5$ \\
\hline
\end{tabular}

Data are presented as mean \pm SD or as absolute numbers (\%) 


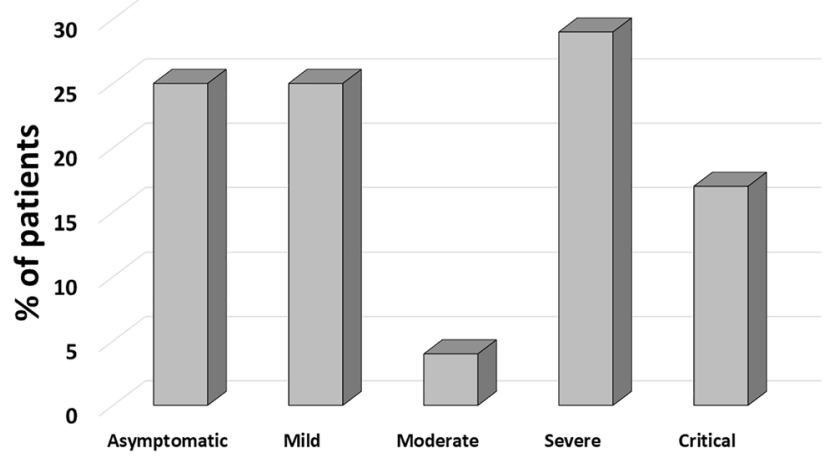

Fig. 2 Maximum COVID-19 severity. Six patients (25\%) were asymptomatic throughout COVID-19 infection, 7 were classified as mild-moderate disease (29\%), 7 were classified as severe and 4 (17\%) as critical COVID-19 infection

including higher mean C-reactive protein levels $(9 \pm 6.4$ vs. $3.6 \pm 3.3 \mathrm{mg} / \mathrm{dL}, \mathrm{p}=0.041)$ and lower mean serum albumin $(3.1 \pm 0.4$ vs. $3.7 \pm 0.2 \mathrm{~g} / \mathrm{dl}, \mathrm{p}=0.013)$ compared to subjects with moderate or milder COVID-19. Whole genome sequencing confirmed all 23 viral samples from patients diagnosed with RT-PCR as SARS-CoV-2 variant B.1.617.2 (delta variant).

Regarding COVID-19 infection sources, 6/24 cases (25\%) were identified as nosocomial, acquired during recent hospitalizations for causes unrelated to COVID-19. Two cases (8\%) occurred in subjects living in chronic care facilities. One subject, a health-care professional, acquired COVID-19 while working in an inpatient rehabilitation center.

Among patients who acquired nosocomial COVID-19, $5 / 6(83.3 \%)$ developed severe or critical illness, 2 died as a result of COVID-19, and 2 remained oxygen dependent for over a month after hospital discharge..

Thirteen (54\%) MHD patients were hospitalized due to COVID-19. COVID-19-directed treatment included dexamethasone for $13(54 \%)$, remdesivir in 1 (4\%) and tocilizumab in 2 patients $(8 \%)$. None of the patients received therapy with monoclonal antibodies, convalescent plasma or baricitinib. Six patients (25\%) received antibiotics for presumed or definite bacterial co-infection.

\section{Mortality}

The 30-day mortality rate after acquiring breakthrough COVID-19 was $21 \%$ (5/24 patients). Compared with vaccinated MHD patients who did not develop COVID-19, overall mortality during the 4-month study period was significantly higher for those who had breakthrough COVID-19 (3/91 $(3.3 \%)$ vs. $5 / 24(21 \%)$, respectively; $p=0.003)$. Odds ratio for all-cause mortality was 7.6 (95\% CI 1.4-41, p=0.002) for the study group compared to controls. Survival curves according to COVID-19 are shown in Fig. 3.

Predictors for all-cause mortality during the study period include heart failure and COVID-19 infection. However, COVID-19 remained the most important predictor of mortality in the multivariate logistic regression analysis model (Table 4).

\section{Discussion}

This study reports on 24 cases of breakthrough COVID-19 among fully vaccinated MHD patients, caused by SARSCoV-2 variant B.1.617.2, 6 months or more after vaccination. The risk for breakthrough COVID-19 was associated
Fig. 3 Kaplan-Meier survival curve from 1 June, 2021 $(\mathrm{p}=0.002)$

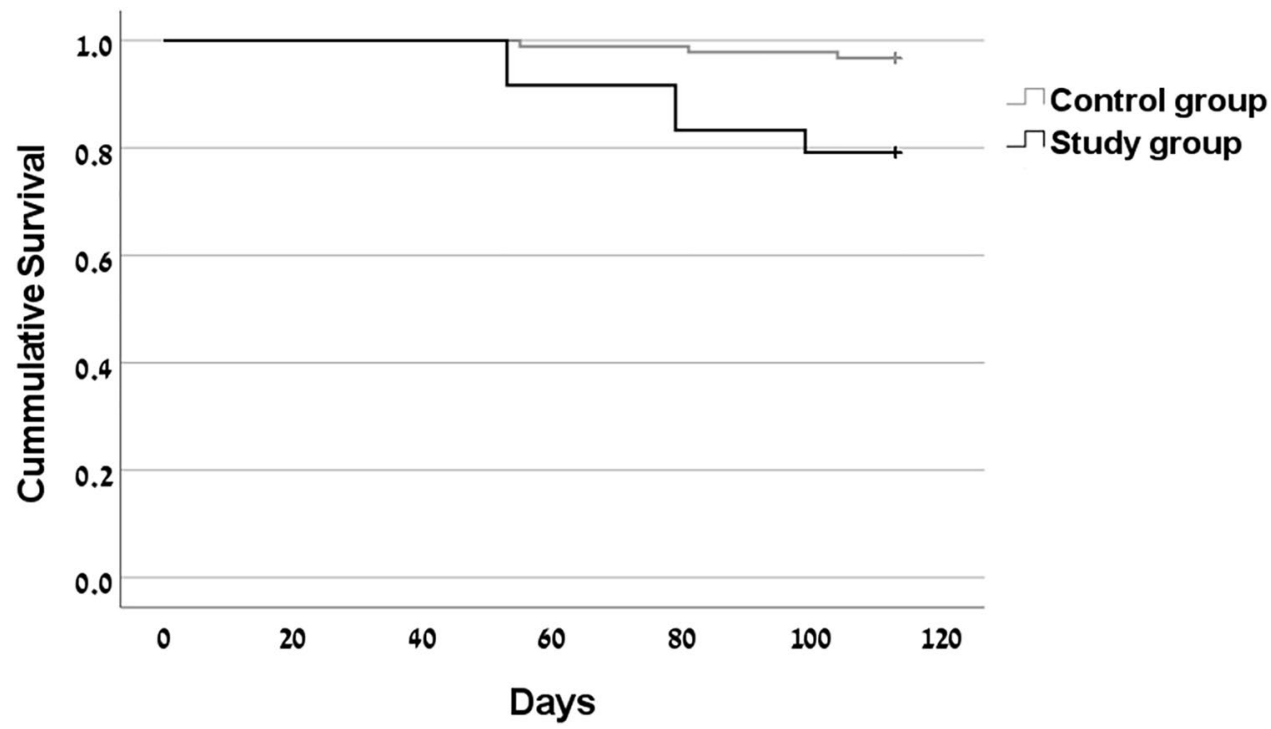


Table 4 Multivariate logistic regression analysis model for predictors of mortality

\begin{tabular}{lllll}
\hline Variable & Odds ratio & \multicolumn{2}{l}{$\begin{array}{l}\text { 95\% confidence } \\
\text { interval }\end{array}$} & p value \\
\cline { 3 - 4 } & & Lower & Upper & \\
\hline COVID-19 infection & 7.6 & 1.4 & 41 & $\mathbf{0 . 0 2}$ \\
Diabetes mellitus & 0.6 & 0.1 & 3.4 & 0.6 \\
Ischemic heart disease & 0.6 & 0.1 & 3.7 & 0.6 \\
Heart failure & 7.4 & 1 & 54 & $\mathbf{0 . 0 5}$ \\
\hline
\end{tabular}

Bold values represent statistical significance

with lower IgG anti-S levels and immunosuppression. Most cases were symptomatic, over $50 \%$ required hospitalization for COVID-19, and 46\% developed severe or critical disease, which required specific therapy. Breakthrough infection led to a significant mortality rate of $21 \%$ of patients. A minority of cases were of nosocomial origin $(25 \%)$ or otherwise healthcare-associated (totaling 9/24, 37.5\%).

The efficacy of mRNA-based vaccines, including BNT162b2, against COVID-19, and especially against severe disease is remarkably high. However, this efficacy gradually declines over time $[1,11]$. The B.1.617.2 variant became increasingly dominant worldwide beginning in April 2021, and accounted for over $90 \%$ of sequenced viruses in many countries during August-September 2021 [12]. Full 2-dose vaccination with BNT162b2 seemed to retain high effectiveness against the B.1.617.2 variant in some studies [13]. However, several studies reported significantly reduced vaccine effectiveness, ranging from 53 to $66 \%$, at the time when the delta variant became predominant [14, 15]. It has been suggested that this decline in vaccine effectiveness may result from the combined effect of waning immunity over time and the concomitant surge of the B.1.617.2 variant [16].

In addition to increased transmissibility, the delta variant is also associated with more severe disease in unvaccinated subjects than with previously dominant variants, leading to higher risk of hospital admissions for COVID-19 [17].

Our results are in agreement with previous observations. The risk for breakthrough COVID-19 caused by the B.1.617.2 variant was not negligible in our cohort of vaccinated MHD patients, 6 months after vaccination. Humoral response to the BNT162b2 vaccine is weaker in MHD patients than in healthy controls [18]. Both early and late post-vaccination IgG anti-S levels are lower in older and immunocompromised MHD patients [7, 8, 19, 20]. In our cohort, breakthrough COVID-19 was associated with immunosuppression. Subjects with breakthrough infection had lower IgG anti-S levels than controls. This might be the result of waning immunity since vaccination, or an initial weaker response to the vaccine, or both. Antibody levels increase substantially in MHD patients after a third dose of the BNT162b2 vaccine [21, and unpublished data]. Increased antibody responses with improved neutralization kinetics, including against the delta variant, were recently reported [22]. A third (booster) dose of the vaccine demonstrated effectiveness in reducing confirmed COVID-19 and severe disease in a large study from Israel [23], during the B.1.617.2 variant predominance [11].

Nosocomial COVID-19 cases are a specific concern in MHD patients. Hospital-acquired infection is a risk-factor for worse COVID-19 outcomes [24]. An outbreak of the delta variant, which included breakthrough infections in vaccinated healthcare workers and admitted patients, was recently reported. It resulted in significant morbidity, although mortality was limited to hospitalized, mostly unvaccinated patients [25]. Another recent study reported on a nosocomial outbreak of the delta variant among a highly-vaccinated group of patients and healthcare workers (96.2\% of the exposed population was fully vaccinated with 2 doses of BNT162b2). Again, severe and critical COVID19 , as well as fatality rates, were significant among admitted patients. The time from vaccination in this report was at least 5 months, implying that waning immunity was a major contributor [26]. In both reports, SARS-CoV-2 transmission occurred despite wearing surgical masks [25, 26]. Our study adds to the concerns of healthcare-associated COVID-19 among vaccinated individuals.

The main limitations of the study include its retrospective nature and modest sample size. Viral genomic sequencing was not performed in the case who was diagnosed with COVID-19 based on anti-N-Ab conversion. Nevertheless, B.1.617.2 became the dominant variant in Israel during the study period, accounting for 93-99\% of cases in which viral genomes were sequenced nationwide, during July-August 2021 [11], and $100 \%$ of cases sequenced from our hospital. Thus, it is highly likely that this case was also caused by the delta variant. IgG anti-S levels were available only 6 months following vaccination and for only $29 \%$ of the cases with breakthrough infection, which could have biased the results. However, these patients were otherwise similar to the rest of the study group, and as such we believe they are an adequate, representative sample. We used a cutoff of $50 \mathrm{AU} / \mathrm{ml}$ as reflecting adequate levels of IgG anti-S-Ab; however, the protective threshold is currently undefined.

\section{Conclusions}

Even vaccinated MHD patients may be at risk for COVID-19 caused by the B.1.617.2 variant, which can have dire outcomes. Immunocompromised patients and those who have a weaker immune response to the vaccine are more prone to breakthrough infections, which may occur in healthcare settings. 
Utilizing precautionary measures to prevent virus transmission during hospitalizations should be mandatory even among populations with high vaccine coverage. Prioritizing MHD patients for a third or eventually further vaccine dose may be warranted, pending further evidence.

Supplementary Information The online version contains supplementary material available at https://doi.org/10.1007/s40620-022-01245-9.

Acknowledgements We thank Faye Schreiber, MS (Meir Medical Center) for editing the manuscript.

Author contributions Research area and study design: OW, NN, SB, $\mathrm{PS}, \mathrm{KCH}$; data acquisition: $\mathrm{OW}, \mathrm{NN}, \mathrm{DE}, \mathrm{AF}, \mathrm{KCH}$; data analysis and interpretation: $\mathrm{OW}, \mathrm{MS}, \mathrm{AG}, \mathrm{PA}, \mathrm{SB}, \mathrm{KCH}$; statistical analysis: $\mathrm{OW}$, $\mathrm{KCH}$; supervision or mentorship: $\mathrm{SB}, \mathrm{KCH}$.

Funding The authors declare that they have no relevant financial interests.

\section{Declarations}

Conflict of interest The authors declare that they have no competing financial or other interests or personal relationships that could have influenced this manuscript.

Ethical approval The study was approved by the Ethics Committee and Institutional Review Board of Meir Medical Center (application no. MMC-0016-21). The committee waived the requirement for informed consent due to the retrospective nature of the study. The study was performed in accordance with the Declaration of Helsinki and Good Clinical Practice guidelines.

\section{References}

1. Thomas SJ, Moreira ED Jr, Kitchin N, Absalon J, Gurtman A, Lockhart S, Perez JL, Pérez Marc G, Polack FP, Zerbini C, Bailey R, Swanson KA, Xu X, Roychoudhury S, Koury K, Bouguermouh S, Kalina WV, Cooper D, Frenck RW Jr, Hammitt LL, Türeci Ö, Nell H, Schaefer A, Ünal S, Yang Q, Liberator P, Tresnan DB, Mather S, Dormitzer PR, Sahin U, Gruber WC, Jansen KU, C4591001 Clinical Trial Group (2021) Safety and efficacy of the BNT162b2 mRNA Covid-19 vaccine through 6 months. N Engl J Med. https://doi.org/10.1056/NEJMoa2110345 (Epub ahead of print)

2. Bergwerk M, Gonen T, Lustig Y, Amit S, Lipsitch M, Cohen C, Mandelboim M, Levin EG, Rubin C, Indenbaum V, Tal I, Zavi$\tan$ M, Zuckerman N, Bar-Chaim A, Kreiss Y, Regev-Yochay G (2021) Covid-19 breakthrough infections in vaccinated health care workers. N Engl J Med. https://doi.org/10.1056/NEJMoa2109072 (Epub ahead of print)

3. Hacisuleyman E, Hale C, Saito Y, Blachere NE, Bergh M, Conlon EG, Schaefer-Babajew DJ, DaSilva J, Muecksch F, Gaebler C, Lifton R, Nussenzweig MC, Hatziioannou T, Bieniasz PD, Darnell RB (2021) Vaccine breakthrough infections with SARSCoV-2 variants. N Engl J Med 384(23):2212-2218. https://doi. org/10.1056/NEJMoa2105000 (Epub 2021 Apr 21)

4. Tao K, Tzou PL, Nouhin J, Gupta RK, de Oliveira T, Kosakovsky Pond SL, Fera D, Shafer RW (2021) The biological and clinical significance of emerging SARS-CoV-2 variants. Nat Rev Genet. https://doi.org/10.1038/s41576-021-00408-x (Epub ahead of print)

5. Bruchfeld A (2021) The COVID-19 pandemic: consequences for nephrology. Nat Rev Nephrol 17(2):81-82. https://doi.org/10. 1038/s41581-020-00381-4

6. Broseta JJ, Rodríguez-Espinosa D, Rodríguez N, Mosquera MDM, Marcos MÁ, Egri N, Pascal M, Soruco E, Bedini JL, Bayés B, Maduell F (2021) Humoral and cellular responses to mRNA-1273 and BNT162b2 SARS-CoV-2 vaccines administered to hemodialysis patients. Am J Kidney Dis 78(4):571-581. https://doi.org/ 10.1053/j.ajkd.2021.06.002 (Epub 2021 Jun 24)

7. Grupper A, Sharon N, Finn T, Cohen R, Israel M, Agbaria A, Rechavi Y, Schwartz IF, Schwartz D, Lellouch Y, Shashar M (2021) Humoral response to the Pfizer BNT162b2 vaccine in patients undergoing maintenance hemodialysis. Clin J Am Soc Nephrol. https://doi.org/10.2215/CJN.03500321 (Epub ahead of print)

8. Nacasch N, Erez D, Lishner M, Benchetrit S, Rozenberg I, Sarel E, Shitrit P, Wand O, Cohen-Hagai K (2021) Long-term antibody response to the BNT162b2 vaccine among maintenance hemodialysis patients. Am J Kid Dis. https://doi.org/10.1053/j.ajkd.2021. 09.002 (in press)

9. Zuckerman NS, Pando R, Bucris E, Drori Y, Lustig Y, Erster O, Mor O, Mendelson E, Mandelboim M (2020) Comprehensive analyses of SARS-CoV-2 transmission in a public health virology laboratory. Viruses 12(8):854. https://doi.org/10.3390/v12080854

10. COVID-19 Treatment Guidelines Panel (2021) Coronavirus disease 2019 (COVID-19) treatment guidelines. National Institutes of Health. Available at https://www.covid19treatmentguidelines. nih.gov/. Accessed 29 July 2021

11. Rosenberg ES, Holtgrave DR, Dorabawila V, Conroy M, Greene D, Lutterloh E, Backenson B, Hoefer D, Morne J, Bauer U, Zucker HA (2021) New COVID-19 cases and hospitalizations among adults, by vaccination status-New York, May 3-July 25, 2021. MMWR Morb Mortal Wkly Rep 70(34):1150-1155. https://doi. org/10.15585/mmwr.mm7034e1

12. Our World in Data (2021) SARS-CoV-2 variants in analyzed sequences, Israel. Available at https://ourworldindata.org/graph er/covid-variants-area?country= ISR. Accessed 1 Oct 2021

13. Lopez Bernal J, Andrews N, Gower C, Gallagher E, Simmons R, Thelwall S, Stowe J, Tessier E, Groves N, Dabrera G, Myers R, Campbell CNJ, Amirthalingam G, Edmunds M, Zambon M, Brown KE, Hopkins S, Chand M, Ramsay M (2021) Effectiveness of Covid-19 vaccines against the B.1.617.2 (Delta) variant. N Engl J Med 385(7):585-594. https://doi.org/10.1056/NEJMoa2108891 (Epub 2021 Jul 21)

14. Nanduri S, Pilishvili T, Derado G, Soe MM, Dollard P, Wu H, Li Q, Bagchi S, Dubendris H, Link-Gelles R, Jernigan JA, Budnitz D, Bell J, Benin A, Shang N, Edwards JR, Verani JR, Schrag SJ (2021) Effectiveness of Pfizer-BioNTech and Moderna vaccines in preventing SARS-CoV-2 infection among nursing home residents before and during widespread circulation of the SARSCoV-2 B.1.617.2 (Delta) variant-National Healthcare Safety Network, March 1-August 1, 2021. MMWR Morb Mortal Wkly Rep 70(34):1163-1166. https://doi.org/10.15585/mmwr.mm703 $4 \mathrm{e} 3$

15. Fowlkes A, Gaglani M, Groover K, Thiese MS, Tyner H, Ellingson K, HEROES-RECOVER Cohorts (2021) Effectiveness of COVID-19 vaccines in preventing SARS-CoV-2 infection among frontline workers before and during B.1.617.2 (Delta) variant predominance-eight US Locations, December 2020-August 2021. MMWR Morb Mortal Wkly Rep 70(34):1167-1169. https://doi. org/10.15585/mmwr.mm7034e4

16. Keehner J, Horton LE, Binkin NJ, Laurent LC, Pride D, Longhurst CA, Abeles SR, Torriani FJ (2021) Resurgence of SARS-CoV-2 infection in a highly vaccinated health system workforce. N Engl 
J Med. https://doi.org/10.1056/NEJMc2112981 (Epub ahead of print)

17. Twohig KA, Nyberg T, Zaidi A, Thelwall S, Sinnathamby MA, Aliabadi S, Seaman SR, Harris RJ, Hope R, Lopez-Bernal J, Gallagher E, Charlett A, De Angelis D, Presanis AM, Dabrera G, COVID-19 Genomics UK (COG-UK) consortium (2021) Hospital admission and emergency care attendance risk for SARS-CoV-2 delta (B.1.617.2) compared with alpha (B.1.1.7) variants of concern: a cohort study. Lancet Infect Dis. https://doi.org/10.1016/ S1473-3099(21)00475-8 (Epub ahead of print)

18. Speer C, Göth D, Benning L, Buylaert M, Schaier M, Grenz J, Nusshag C, Kälble F, Kreysing M, Reichel P, Töllner M, Hidmark A, Ponath G, Schnitzler P, Zeier M, Suesal C, Morath C, Klein K (2021) Early humoral responses of hemodialysis patients after COVID-19 vaccination with BNT162b2. Clin J Am Soc Nephrol 16(7):1073-1082. https://doi.org/10.2215/CJN.03700321 (Epub ahead of print)

19. Frantzen L, Cavaille G, Thibeaut S, El-Haik Y (2021) Efficacy of the BNT162b2 mRNA Covid-19 vaccine in a hemodialysis cohort. Nephrol Dial Transplant. https://doi.org/10.1093/ndt/gfab1 65 (Epub ahead of print)

20. Longlune N, Nogier MB, Miedougé M, Gabilan C, Cartou C, Seigneuric B, Del Bello A, Marion O, Faguer S, Izopet J, Kamar N (2021) High immunogenicity of a messenger RNA-based vaccine against SARS-CoV-2 in chronic dialysis patients. Nephrol Dial Transplant 36(9):1704-1709. https://doi.org/10.1093/ndt/gfab193

21. Bensouna I, Caudwell V, Kubab S, Acquaviva S, Pardon A, Vittoz N, Bozman DF, Hanafi L, Faucon AL, Housset P (2021) SARSCoV-2 antibody response after a third dose of the BNT162b2 vaccine in patients receiving maintenance hemodialysis or peritoneal dialysis. Am J Kidney Dis. https://doi.org/10.1053/j.ajkd.2021.08. 005 (Epub ahead of print)

22. Falsey AR, Frenck RW Jr, Walsh EE, Kitchin N, Absalon J, Gurtman A, Lockhart S, Bailey R, Swanson KA, Xu X, Koury K, Kalina W, Cooper D, Zou J, Xie X, Xia H, Türeci Ö, Lagkadinou E, Tompkins KR, Shi PY, Jansen KU, Şahin U, Dormitzer PR,
Gruber WC (2021) SARS-CoV-2 neutralization with BNT162b2 vaccine dose 3. N Engl J Med. https://doi.org/10.1056/NEJMc 2113468 (Epub ahead of print)

23. Bar-On YM, Goldberg Y, Mandel M, Bodenheimer O, Freedman L, Kalkstein N, Mizrahi B, Alroy-Preis S, Ash N, Milo R, Huppert A (2021) Protection of BNT162b2 vaccine booster against Covid-19 in Israel. N Engl J Med. https://doi.org/10.1056/NEJMo a2114255 (Epub ahead of print)

24. Gupta RK, Harrison EM, Ho A, Docherty AB, Knight SR, van Smeden M, Abubakar I, Lipman M, Quartagno M, Pius R, Buchan I, Carson G, Drake TM, Dunning J, Fairfield CJ, Gamble C, Green CA, Halpin S, Hardwick HE, Holden KA, Horby PW, Jackson C, Mclean KA, Merson L, Nguyen-Van-Tam JS, Norman L, Olliaro PL, Pritchard MG, Russell CD, Scott-Brown J, Shaw CA, Sheikh A, Solomon T, Sudlow C, Swann OV, Turtle L, Openshaw PJM, Baillie JK, Semple MG, Noursadeghi M, ISARIC4C Investigators (2021) Development and validation of the ISARIC 4C deterioration model for adults hospitalised with COVID-19: a prospective cohort study. Lancet Respir Med 9(4):349-359. https://doi.org/ 10.1016/S2213-2600(20)30559-2 (Epub 2021 Jan 11)

25. Hetemäki I, Kääriäinen S, Alho P, Mikkola J, Savolainen-Kopra C, Ikonen N, Nohynek H, Lyytikäinen O (2021) An outbreak caused by the SARS-CoV-2 Delta variant (B.1.617.2) in a secondary care hospital in Finland, May 2021. Euro Surveill 26(30):2100636. https://doi.org/10.2807/1560-7917.ES.2021.26.30.2100636

26. Shitrit P, Zuckerman NS, Mor O, Gottesman B, Chowers M (2021) Nosocomial outbreak caused by the SARS-CoV-2 Delta variant in a highly vaccinated population, Israel, July 2021. Euro Surveill 26(39):2100822. https://doi.org/10.2807/1560-7917.ES. 2021.26.39.2100822

Publisher's Note Springer Nature remains neutral with regard to jurisdictional claims in published maps and institutional affiliations. 